\title{
BORE WELL RESCUE ROBOT WITH LIFE, FRACTURE AND DISTANCE DETECTION TECHNIQUE
}

\author{
Girish.S.Malimath ${ }^{1}$ \\ ${ }^{I}$ B.E (E\&CE) in Basavakalyan Engineering College, Basavakalyan
}

\begin{abstract}
In these recent days it's becoming a common tragedy that small kids/humans or even animals fell into the boreholes which are left open and many have took their last breath. A little impediment in the rescue operation of the strucked person can results in the death of a person. To avoid all such disasters we are producing an idea to extricate the strucked human using advanced robo. It is not enough if we just design a robo, it is also essential to detect weather human is alive or not and to keep him alive by providing sufficient amount of oxygen and it is required to measure the distance at which the human is strucked. To provide a proper treatment we are required to detect the fractures, if any. This robo is operated through pc using zigbee.
\end{abstract}

Keywords: Extricate, Robot, Oxygen, Distance Measurement, Detect the Fracture, PC, Zigbee

\section{INTRODUCTION}

The new enormous high speed computers, reliable computer software's along with embedded c offers a new "fit occasion" for fresh robot control/handling and clear apprehension of new method of control theory . Robotics is a field which involves controlling of machines using computer, all these computer controlled machines or devices will execute the operations which require high accuracy or irksome or the work which results with causalities if they are done by the humans[1]. All the improvements in the computer technology, control devices along with improved control algorithms resulted with the enormously high speed, accurate and brilliant robots [2]. in these recent days it becoming a common tragedy that small kids and even adults and sometimes animals fell into the uncovered bore wells. The delay and erogenous human methods made many to breath their last. To overcome all such difficulties we are producing an idea of "Rescue Robot" which will be as small as the width of the borehole in size and extricate strucked person or animals in much professional way so that no harm can be produced to the human or animal body. It will also detects at which distance a human is present and also examine whether he is alive or dead. it also detects for the fractures so that he/she can have proper medication or treatment without delay when he/she is hospitalized along with all these it will also provide the proper amount of oxygen required when there is scarcity of oxygen in the borehole from the external sources[3].

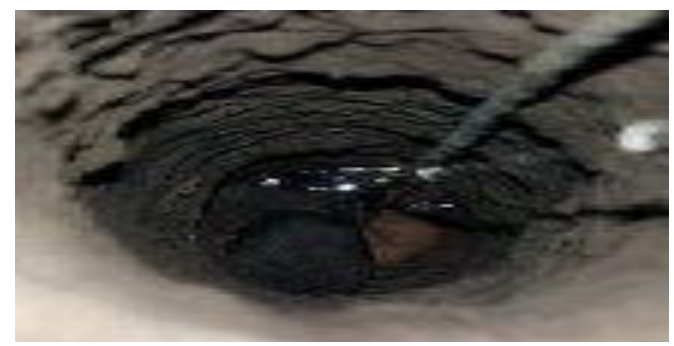

Fig 1 Baby strucked in bore well which is uncovered.

\section{OBJECTIVES OF THE PAPER}

1. Rescuing human with no harm.

2. Proper quick and proper medication after rescue of strucked person

3. Controlling Robot wirelessly using zigbee.

4. Implementation of pick and place concept to a Robot.

5. Audio and Video inspection of rescue operation.

\section{EXISTING MODULE}

The below mentioned figure 2 explains about the existing technology which has disadvantages which are mentioned next to the block diagram in fig 2 .

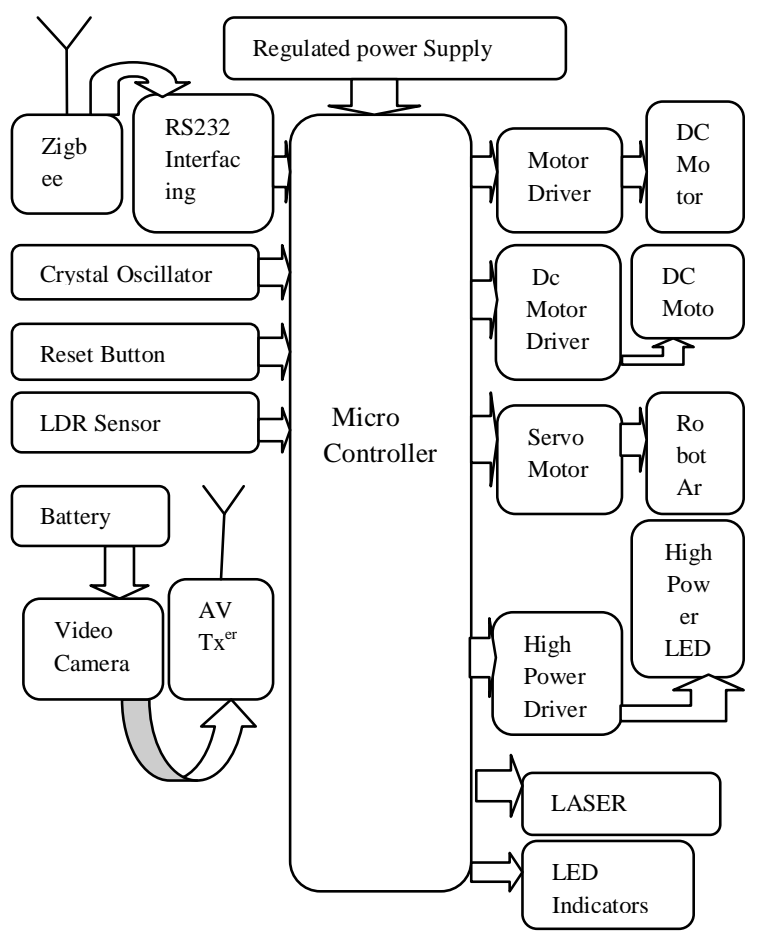

Fig 2 Block diagram of design and construction of rescue robot and pipeline inspection using zigbee for receiver 


\section{DISADVANTAGES OF EXISTING TECHNOLOGY}

1. It will not gives the information about at which distance or how deep a human or any other animal is strucked.

2. The existing paper will not discuss about the LASER which is used.(considered as failed to use the allotted resources or simply the waist of resource).

3. It will not detect whether or not Human is alive or dead.

4. It has no arrangements in it which really helps in providing proper treatments or medication soon after rescue.

\section{PROPOSED MODEL}

To overcome all the above mentioned Disadvantages we are proposing a new method as mentioned below

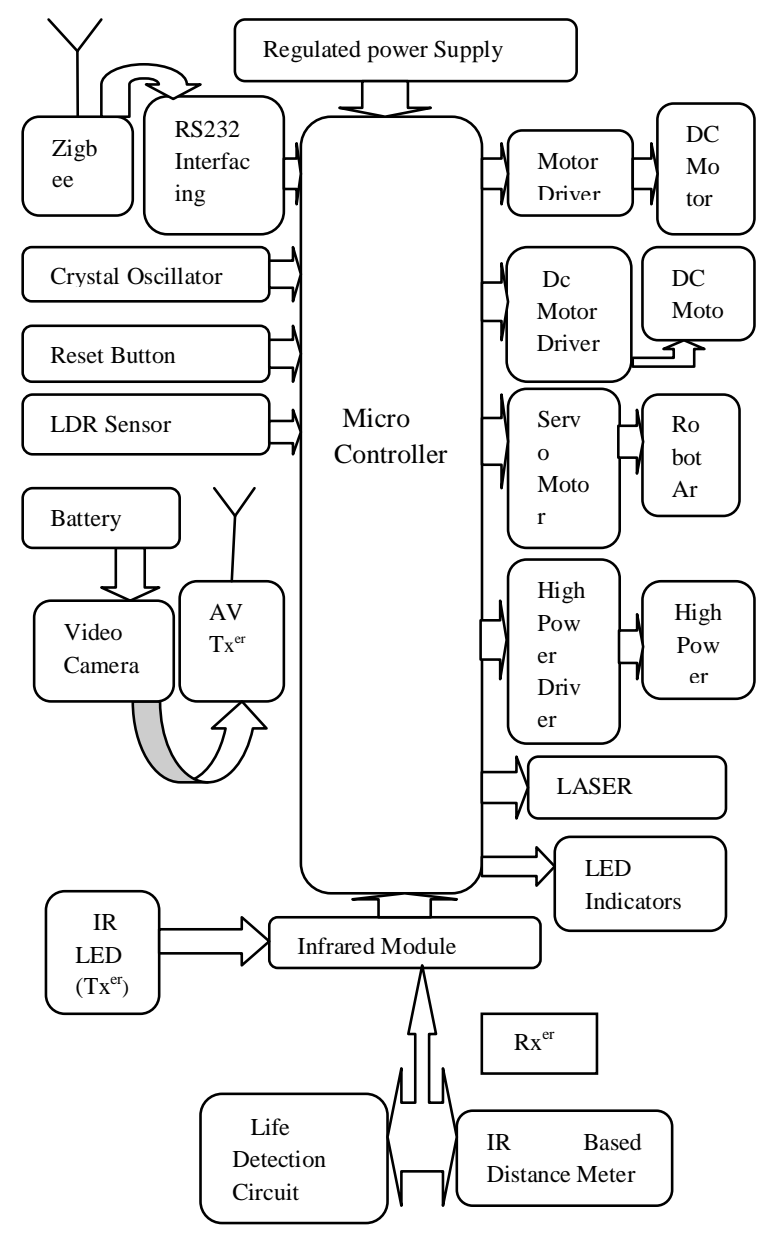

Fig 3 Bore well Rescue Robot with Life, Fracture and Distance Detection Technique.

Description: This paper discusses about the robot which will be completely controlled by the pc(user) with the use of IEEE 802.15.4 Zigbee std. We can inspect the operation of the robot i.e., rescue robot using wireless camera this enables the users to view both audio and video on the television set or even on pc if it has TV Tuner Card.

\subsection{High Power LED}

These LED's are used as a light source when visibility in the bore well becomes weak.

\subsection{Zigbee}

Zigbee is a WPAN technology based on IEEE802.15.4 std. Here we have preference to Zigbee over Bluetooth and WIFI because of the reason that it is intelligent enough to form a mesh network between nodes. Meshing is more or less similar to daisy chaining where computers, peripheral devices are connected one after the other, this is helpful in covering large network range area . Zigbee enables the user to transmit the data/information from the keyboard of the computer.

\subsection{LDR}

The term LDR stands for light dependent register, this a kind of sensor which will send the information over the Zigbee to the PC to turn on the High Source LED.

\subsection{Camera}

The camera which is used here is wireless in nature. This will transmit the live recording of both audio and video using a AV Transmitter which can be viewed on the Television or computer if it supports TV tuner card.

\subsection{Microcontroller (AT32UC3L0256)}

A Microcontroller is a computer which is meant for specific task i.e., dedicated to single task and run only one program at a time. It has single integrated circuit which contains central processing unit (CPU) which is united with a system support function such as oscillator, ADC, Serial Ports, I/O Ports, Timers/Counters, Watchdog timer. For this rescue robot module which is proposed we are using AT32UC3L0256 Controller. It supports RISC instruction along with the DSP Instructions. It has a memory protection unit(MPU) which is a secure access unit(SAU) providing user defined peripheral protection. Here 32 in the name of the controller indicates that it of 32 bit controller and 256 indicates that 256 bits of internal Flash memory. It has external interrupt controller(EIC) which allows pins to be configured as external interrupts. Each external interrupt has its own interrupt request which can be masked individually.

\subsection{Motors}

\subsubsection{Motor}

DC motor is such a device which generates mechanical energy using electrical energy typically by the method of interaction of magnetic fields and current carrying conductors. To produce a torque as output we have to give the input supply either as current or as a voltage. There are two major ports in DC motors as 1)Armature which rotates and 2) Field coils which are static in nature. 


\subsubsection{Servo Motor}

Servo motor is used to operate the arms of the robot. The servo motor is an assembly of 4 things which are as follows: 1) A normal DC motor 2) A gear reduction unit 3)A position sensing device(usually a potentiometer-a control volume knob). and 4) A control circuit. The function of the servo motor is to receive the control signals that represents a desired output position of the servo shaft. It uses a rotational sensing device with this it will come to know in which direction motor must turn to move.

\subsection{IR Transmitter}

Here LED is used as a transmitter. there are three types of IR LED's are present namely 1 . Small Power LED(1mw10mw) 2. Medium Power LED(10mw-50mw) 3. Large Power LED(50mw-100mw). For our rescue robot module Large Power LED's are the best choice. These will transmit the light with longer wavelength which are invisible to human eyes to detect the object/person .

\subsection{IR Receiver}

\subsubsection{IR Based Distance Meter}

This is constructed in such a way that it will recognize the human beings by the emission of infrared rays with the range of 5-15 lumens. An IR tx ${ }^{\mathrm{er}}$ consists of an IR LED with a series resistance where as at the receiver it consists of a photo diode which receives reflected IR signals. The voltage obtained from the reelected signal is in between it's max and min operating voltage and that will be fed to microcontroller for further operations.

\subsubsection{Life Detection Circuit}

The main objective of this life detection circuit is to find out the change in the concentration of the blood at the tips of the fingers. If it changes, it is considered as human is alive else dead. This can be achieved using LASAER source and LDR.

\subsection{Laser/Fracture Detection Unit}

It can be accomplished by sending the LASER light towards objects/humans using 2 or more different wavelengths and it can be detected by LASER beams which are reflected back towards the photo detector from the objet

\section{CONCLUSION}

The use of advanced IC's ensures that it is capable of meeting all the results which are mentioned in entire paper moreover it will become an advanced and highly professional solution in the field of rescue operations it will replaces all the erogenous human technology which may cause the harm to the victim.

\section{FUTURE SCOPE}

1. By connecting the sensors like ECG etc.. on the robot we can make it as mobile laboratory which will be very much helpful in providing quick medication to the victims

2. By connecting temperature and smoke sensor we can get the information regarding smoke and temperatures in the danger zones on our personnel computer itself.

\section{REFERENCES}

[1]. Shukla Shubhendu S, Jaiswal Vijay, Applicability of Artificial Intelligence in Different Fields of Life, International Journal of Scientific Engineering and Research, pp. 28-35, 2013.

[2]. Birk, A and Carpin,S., 2006., Rescue Robotics - a crucial milestone on the road to autonomous systems., Advanced Robotics Journal., 20(5)., pp 1-11.

[3]. Ashutosh Gupta and Bethy Thomos, A new Revolutionary Infrared Life Detection system using ATMEGA168, International Journal of Embedded System and Application(IJSEA) vol. 2, no. 3, September 2012. 